\title{
A retrospective study on group B beta haemolytic streptococcal sepsis in early infancy
}

\author{
H M K N Hathlahawatta ${ }^{1}$, D K Samarage ${ }^{2}$, G S H.Liyanage ${ }^{2}$, S Chandrasiri $^{3}$
}

Sri Lanka Journal of Child Health, 2009; 38: 124-126

(Key words: group B haemolytic streptococcal sepsis, GBS, early infancy)

\begin{abstract}
Objectives To determine the incidence, clinical presentation and outcome of group B haemolytic streptococcal (GBS) sepsis

Design, setting and method This was a two year retrospective observational analysis at Colombo South Teaching hospital. All clinically suspected and bacteriologically confirmed cases of GBS sepsis in infants up to three month of age were analysed. Data was obtained from individual clinical notes and records at the Department of Microbiology.
\end{abstract}

Results There were 21 cases, an incidence of 1.06 per 1000 live births. Thirteen (62\%) were male. Seven (33\%) were premature. Thirteen (62\%) were early onset and $08(38 \%)$ were late onset. Main presenting features on admission were respiratory $11(52 \%)$, poor activity/feeding 09 (43\%) and fever 08 (38\%). Complications were mainly neurological (48\%) Mortality was $4.7 \%$.

\section{Introduction}

Group B beta haemolytic streptococcal (GBS) infection in early infancy is a serious disease with significant morbidity and mortality ${ }^{1,2,3}$. Incidence and prevalence of GBS disease vary among institutions and countries ${ }^{3,4,5,6}$. A significant reduction of GBS infection has been observed in many studies after introduction of guidelines for prevention ${ }^{3,4,7,8}$. However, studies on GBS infection are scarce in Sri Lanka. In our institute during 2007, we observed a considerable number of cases with GBS, having a serious course of illness with complications. This observation prompted us to proceed with this study.

${ }^{1}$ Senior Registrar, Colombo South Teaching Hospital, Kalubowila, ${ }^{2}$ Senior Lecturer, Department of Paediatrics, University of Sri Jayawardenapura, ${ }^{3}$ Consultant Microbiologist, Colombo South Teaching Hospital, Kalubowila

(Received 24 November 2008. Accepted 20 January 2009)

\section{Method}

This study was a retrospective observational analysis over a period of 2 years from January 2006 to December 2007. The study population comprised all infants below three month of age with clinically suspected and bacteriologically confirmed GBS sepsis. All neonatal and paediatric units in Colombo South Teaching hospital (CSTH) were included in this study after obtaining permission from the respective consultant paediatricians. Cases were primarily identified from the special record for GBS culture positive cases at the Department of Microbiology, CSTH. Bed Head Tickets were traced from Record room and the data was collected to a data sheet which included personal data, perinatal and birth history, clinical presentation, investigations, treatment and complications during hospitalised period.

\section{Results}

There were 21 infants under three month of age with clinically suspected and bacteriologically confirmed GBS sepsis. Thus, the unit incidence was 1.06 per 1000 live births. Case categorization according to gender, mode of delivery, gestational maturity, and onset of disease is shown in table 1 .

Table 1

Case categorization

\begin{tabular}{|c|c|}
\hline Category & Number (\%) \\
\hline $\begin{array}{cl}\text { Gender } & \\
\bullet & \text { Male } \\
\bullet & \text { Female }\end{array}$ & $\begin{array}{l}13(62) \\
08(38)\end{array}$ \\
\hline $\begin{array}{cc}\text { Mode of delivery } \\
\bullet \quad \text { Vaginal } \\
\bullet \quad \text { Caesarean }\end{array}$ & $\begin{array}{l}13(62) \\
08(38)\end{array}$ \\
\hline $\begin{array}{cl}\text { Maturity } & \\
\bullet & \text { Term } \\
\bullet & \text { Preterm }\end{array}$ & $\begin{array}{l}14(67) \\
07(33)\end{array}$ \\
\hline $\begin{aligned} \text { Onset } & \\
\bullet & \text { Early } \\
\bullet & \text { Late }\end{aligned}$ & $\begin{array}{l}13(62) \\
08(38)\end{array}$ \\
\hline
\end{tabular}


Of the 7 preterm babies, six were between 34 to 36 weeks and one was 31 weeks maturity. Of the 13 babies with early onset sepsis, eleven presented within 24 hours of birth and the rest within one week. The 8 late onset babies were admitted at a mean age of 26 days (14-85days). The presenting features of GBS sepsis on admission are shown in Table 2.

Table 2

Presenting features of GBS sepsis

\begin{tabular}{|l|c|}
\hline Presenting future & Number (\%) \\
\hline Respiratory dysfunction & $11(52)$ \\
\hline Poor activity /feeding & $09(43)$ \\
\hline Fever & $08(38)$ \\
\hline Jaundice & $02(9.5)$ \\
\hline Other & $02(9.5)$ \\
\hline
\end{tabular}

Respiratory dysfunction consisted of tachypnoea in 10 cases and apnoea in one case. Many had more than one presenting feature. Complications encountered are shown in Table 3.

Table 3

Complications of GBS Sepsis

\begin{tabular}{|c|c|}
\hline Complication & Number (\%) \\
\hline \begin{tabular}{ll}
\multicolumn{2}{l}{ Neurological } \\
- & Meningitis \\
- & Seizures \\
- & Subdural effusion \\
- & Coma
\end{tabular} & $\begin{array}{l}10(48) \\
04(19) \\
02(9.5) \\
01(4.7)\end{array}$ \\
\hline Respiratory & $08(38)$ \\
\hline Gastro-intestinal & 04 (19) \\
\hline Circulatory & $02(9.5)$ \\
\hline Musculoskeletal & $02(9.5)$ \\
\hline
\end{tabular}

Whilst blood cultures were positive in all 21 cases, CSF cultures were uniformly negative. There was one death in this series giving a mortality of $4.7 \%$. There was a rare situation of twins presenting with the disease at different ages. One twin presented with GBS sepsis and meningitis at the age of 35 days. The other twin presented with GBS meningitis at the age of 85 days and underwent neurosurgery for subdural effusions (Figure 1).

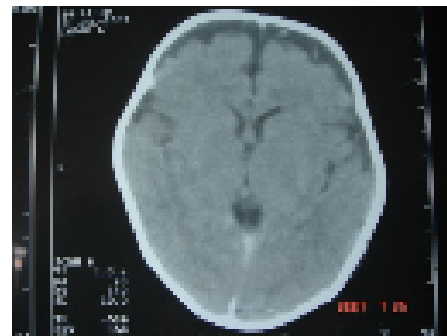

Figure 1: CT scan of brain showing bifrontal subdural effusions

\section{Discussion}

GBS infection is a popular area for medical research throughout the world. In fact, many centres are currently looking at the outcome of preventive strategies ${ }^{2,3,6,7}$. However, there are only a few studies published in Sri Lanka related to this topic.

During this study, we focused on proven cases of GBS sepsis with positive blood cultures. CSF cultures were negative in these cases, probably as lumbar punctures were done after initiation of antibiotics. In our series the incidence of GBS disease was 1.06 per 1000 live births which is slightly higher than in a recently published study (0.82) in Sri Lanka ${ }^{1}$. However, in many other countries, before initiation of firm prophylactic measures, the incidence was slightly more than 1 per 1000 live births, but most of the institutes achieved significant $(>50 \%)$ reduction after preventive strategies $^{2.3 .7}$. It is very likely that the real burden of disease is underestimated in most studies owing to the high incidence of culture-negative sepsis possibly because of antibiotic administration to the mother $^{3,8}$. Additionally, the sensitivity of a single blood culture is a concern where collection of blood for culture in a neonate is usually a difficult task.

Majority (62\%) of cases in our study were boys, a finding similar to that in many studies ${ }^{1,2,4,9}$. Male sex is generally considered as a risk factor for GBS sepsis. Analysis of mode of delivery revealed that the majority were normal vaginal deliveries $(62 \%)$. In this series $07(33 \%)$ cases were premature deliveries $(<36$ weeks), which is similar to available local data $(32 \%)^{1}$. However, many overseas studies show higher percentage of preterm cases, thus defining prematurity as a significant risk factor for this infection ${ }^{3,10}$.

In this study, special attention was given to clinical presentation and complications of GBS disease. Respiratory dysfunction was the commonest presenting feature $(52 \%)$ followed by poor activity / poor feeding (43\%). Fever as a presenting feature was noted only in $38 \%$ cases. There were 2 cases with unusual musculoskeletal signs on presentation, one baby presenting with features of septic arthritis and the other with dactylitis.

Analysis of complications in this series revealed a higher incidence of meningitis and its sequelae $(47.6 \%)$. Two out of 10 babies with meningitis developed subdural effusions needing neurosurgical intervention. One baby got multiple seizures and was comatose for almost a week. Subsequent review of this baby at 6 months revealed features of severe cerebral damage with quadriplegia, refractory epilepsy 
and cortical blindness. Various degrees of long term neurological insults can occur with neonatal meningitis ranging from severe cerebral palsy to subtle neurobehavioural and cognitive dysfunction ${ }^{2,3,8}$. The mortality rate of $4.7 \%$ in this study is slightly higher than the study done by $\mathrm{S}$ Wanigathunga et al. $(3.6 \%)^{1}$. But many other overseas studies show case fatality rates of $3-5 \%^{2,7,9}$. However, there is a high possibility for underestimating the true mortality due to culture negative GBS sepsis ${ }^{3,8}$.

There were several limitations to this study. Unavailability of the laboratory facility for CSF bacterial antigen test was one such matter. It would have been possible to detect more cases with this test, as the CSF cultures are usually negative due to the administration of antibiotics prior to lumbar puncture. In this study we could not analyse the category of possible GBS disease (positive surface swab cultures but negative blood cultures) due to the insufficient and ill defined clinical and laboratory data.

\section{Conclusions}

- GBS sepsis is a serious disease in our neonatal and paediatric units.

- The high possibility for meningitis and its sequelae highlights the need for aggressive preventive and interventional measures.

\section{Acknowledgements}

We thank the Consultant Paediatricians and Director of CSTH for allowing us access to the relevant records.

\section{References}

1. Wanigathunga S, Weerasekara M. Neonatal earlyonset group B streptococcal disease: incidence, clinical presentation and outcome. The $12^{\text {th }}$ Asia Pacific Congress of Paediatrics \& $2^{\text {nd }}$ Asia Pacific Congress of Paediatric Nursing Abstracts March 2007: 1 (1):41-2.
2. Carbonell-Estrany X, Figueras-Aloy J, SalcedoAbizanda S, de la Rosa-Fraile M. Probable earlyonset group B streptococcal neonatal sepsis: a serious clinical condition related to intrauterine infection Arch. Dis. Child. Fetal Neonatal Ed. 2008; 93: F79

3. Trijbels-Smeulders M, de Jonge GA, Pasker-de Jong PCM, Gerards LJ Adriaanse AH, et al. Epidemiology of neonatal group B streptococcal disease in the Netherlands before and after introduction of guidelines for prevention. Archives of Diseases in Childhood 2007; 92 (4):271-6.

4. Imelda PU, Carl TD, Marilyn $\mathrm{M}$ et al. Changes in Early-Onset Group B Beta Haemolytic Streptococcal Disease with changing recommendations for prophylaxis. $J$ Perinatal 2002; 22: $516-22$.

5. Centers for Disease Control and Prevention. Prevention of Perinatal Group B Streptococcal Disease. MMWR 2002; 51: 1-18.

6. Nanan RKH, Singh G. Reductions in Incidence of Invasive Group B Streptococcal Disease in the United States JAMA 2008; 300 (14): 1649 - 50.

7. Berardi A, Lugli L, Baronciani D, Creti R, Rossi K. Group B Streptococcal Infections in a Northern Region of Italy. Pediatrics 2007; 120 (3): e487e493.

8. Puopolo KM, Madoff LC, Eichenwald EC. Earlyonset group B streptococcal disease in the era of maternal screening. Pediatrics 2005; 115 (5):12406.

9. Berardi A, Lugli L, Rossi C, Morini MS, Vagnarelli F et al. Group B streptococcus and preventive strategies in Europe. Arch. Dis. Child. Fetal Neonatal Ed. 2008; 93 (3): F249 - F249.

10. Oddie S, Embleton ND. Risk factors for early onset neonatal group B streptococcal sepsis: case control study. BMJ 2002; 325: 308. 